\title{
LOW OHMIC LOSS CYLINDRICAL RADIAL SUPERLATTICE CONDUCTORS USING ALTERNATINGLY ELECTROPLATED MAGNETIC/NONMAGNETIC THIN FILMS FOR MICROWAVE APPLICATIONS
}

\author{
Arian Rahimi , and Yong-Kyu 'YK' Yoon \\ University of Florida, Gainesville, Florida, USA
}

\begin{abstract}
In this work, the first experimental demonstration of the Cylindrical Radial Superlattice (CRS) conductors made of alternating magnetic/nonmagnetic layers grown on a radial conductor operating in the microwave range is reported. The CRS structure has been employed to force the high frequency current to flow through the volume of the conductor by suppressing the generated eddy currents inside the conductor which will lead to reduction of the conductor loss. Also, the implementation of the highest Q-factor microwave inductor made of the CRS conductor with a Q-factor of 45 at $18 \mathrm{GHz}$, which is attributed to low conductor and dielectric losses, has been demonstrated.
\end{abstract}

\section{INTRODUCTION}

Copper is widely used as a known low loss conductor in standard microfabrication processes in IC and MEMS industries mainly due to its high conductivity, ease of deposition and relatively low cost. However, in higher frequencies, its high conductivity is not as effective and beneficial as it is in the low frequency because of the skin effect, where most current is confined in the outermost surface of the conductor thus reducing the effective cross-section of the conductor and increasing the ohmic resistance. Therefore, the conductor loss together with the dielectric loss will give rise to the total loss of the systems operating in microwave range. The dielectric loss could be suppressed in some devices by carefully removing the dielectric material and let the electromagnetic waves propagating through air ([1-2]). However, there is not much work done to decrease the conductor loss which is a major part of the RF losses.

A planar superlattice structure where alternating metal/ferromagnetic thin films are deposited vertically on each other was proposed in [3] where the stack layers are utilized to suppress the skin effect and lower the conductor loss. Because of the negative permeability of the ferromagnetic metal layers in frequencies above the magnetic resonance ( $f_{M R}$ ), it would be possible to make the effective permeability of the multilayer consisting of the negative/positive permeability of the ferromagnetic/nonferromagnetic metals close to zero and increase the skin depth. Therefore, the current will be forced to flow through the volume of the conductor where the effective area is increased leading to a considerably lower conductor loss. Although the planar superlattice conductors have proved to decrease the ohmic loss of the conductors in high frequency region, the electromagnetic discontinuity at the edge of the conductor and therefore large fringing effects, limit the eddy current suppression of the planar superlattice conductors in practice and leave room to further reduce the loss.

We have previously presented the Cylindrical Radial Superlattice (CRS) structure in [4] where theoretical and numerical analyses with circular and conformal boundary conditions have been demonstrated for the suppression of skin effects and therefore that of the RF conductor loss. In this work, we are reporting on (1) the first experimental demonstration of the CRS conductors consisting of alternating magnetic/nonmagnetic nanolayers working in the microwave range and (2) the implementation of the highest Q-factor microwave inductor made of the CRS conductor with a Q-factor of 45 at $18 \mathrm{GHz}$, which is attributed to low conductor and dielectric losses.

\section{THEORY AND ANALYSIS}

At higher frequencies, the ohmic loss of the conductors are totally governed by the conductivity $(\sigma)$ and the skin depth $(\delta)$,

$$
\delta=\sqrt{\frac{2}{\omega \sigma}}
$$

Therefore, due to the fact that the skin depth is getting smaller once the frequency increases, the ohmic loss unavoidably keeps rising in a regular conductor. The schematic of the cylindrical radial superlattice (CRS) conductor's cross-section view to suppress the ohmic loss is given in Figure 1. The proposed design is employing the fact that although the permeability of the nonferromagnetic layers is constant with respect to frequency $\left(\mu_{\mathrm{N}}=1\right.$ at all frequencies), that of the ferromagnetic metal layers is a function of the frequency and is negative between the ferromagnetic resonance frequency, $f_{M R}$ and the anti-resonance frequency, $f_{A R}$.

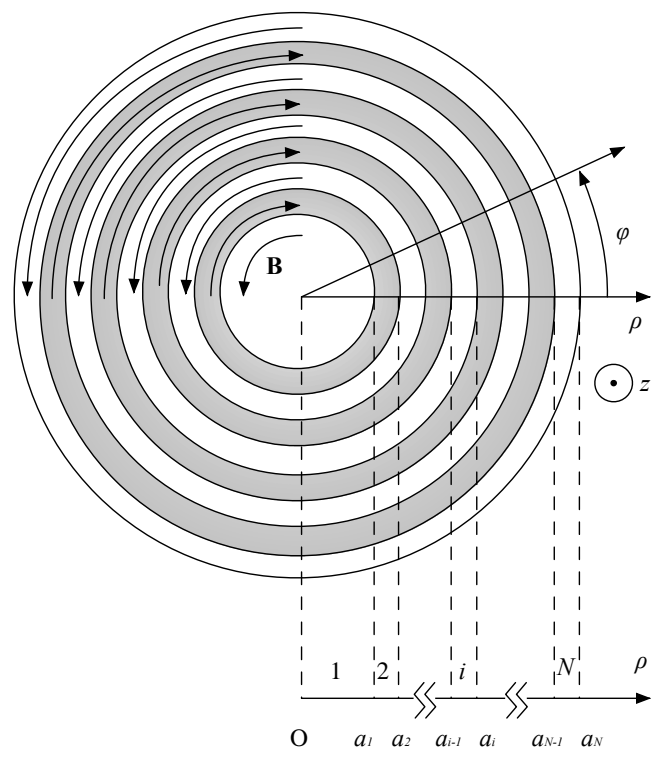

Figure 1: A cylindrical radial superlattice (CRS) conductor composed of metal (white region) and ferromagnetic thin film (grey region) with $N$ layers and total radius $a_{N}$ in the cylindrical coordinate system. The arrows show the direction of the induced eddy currents where opposing each other in the design frequency region due to negative permeability of the ferromagnetic thin film.

By assuming the non-ferromagnetic/ferromagnetic layers as the conductor carrying the high frequency signal, the effective permeability of the stack layers will be given by,

$$
\mu_{\text {eff }}=\frac{\mu_{N} t_{N}+\mu_{F} t_{F}}{t_{N}+t_{F}}
$$

where $\mu_{\mathrm{N}}, \mu_{\mathrm{F}}, \mathrm{t}_{\mathrm{N}}$ and $\mathrm{t}_{\mathrm{F}}$ are the magnetic permeability of the nonferromagnetic layer, the magnetic permeability of the ferromagnetic layer, the thickness of the non-ferromagnetic layer, and the 
thickness of the ferromagnetic layer, respectively.

The dynamic response of magnetic thin films has been investigated theoretically [5] and experimentally [6]. Permalloy $\left(\mathrm{Ni}_{80} \mathrm{Fe}_{20}\right)$ thin films are ones of the most commonly studied soft magnetic materials. Figure 2 shows the calculated permeability of the $\mathrm{Ni}_{80} \mathrm{Fe}_{20}$ using the Landau-Lifshitz-Gilbert (LLG) equation where the magnetic saturation of $\mathrm{M}_{\mathrm{s}}=1 \mathrm{~T}$ has been assumed for the permalloy. The resonance frequency of the permalloy thin film is $\mathrm{f}_{\mathrm{MR}} \approx 900 \mathrm{MHz}$ and the anti-resonance frequency $\mathrm{f}_{\mathrm{AR}} \approx 28 \mathrm{GHz}$.

The device is operating between the ferromagnetic resonance frequency $\left(f_{M R}\right)$ and the anti-resonance frequency $\left(f_{A R}\right)$ where the real part of the permeability, $\mu$ ', is negative (Figure 2). The $r=t_{N} / t_{F}$ which is the ratio of the thickness of the non-ferromagnetic metal layer (usually copper or aluminum) and the ferromagnetic metal layer is an important design parameter which determines the operation frequency.

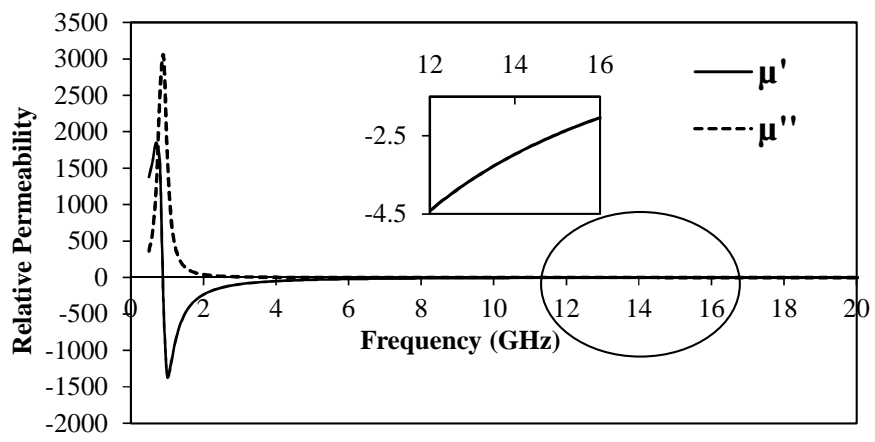

Figure 2: The calculated permeability of the permalloy assuming no external magnetic field. The inset shows the real part $\mu$ ' for the frequency between 12 to $16 \mathrm{GHz}$.

\section{SYSTEM DESIGN AND SIMULATIONS}

In order to verify the performance of the proposed CRS conductors, a CRS superlattice structure has been analyzed and fabricated on a radial conductor. The single turn air-lifted inductors are fabricated on the radial-shape gold wire with a diameter of 28 $\mu \mathrm{m}$. Figure 3 shows the schematic of the CRS inductor where the radial gold wire has been implemented using a commercial wire bonder and one-port coplanar waveguide (CPW) input.

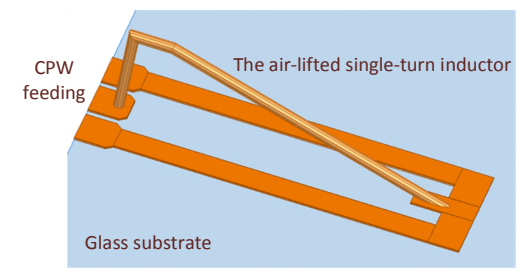

(a)

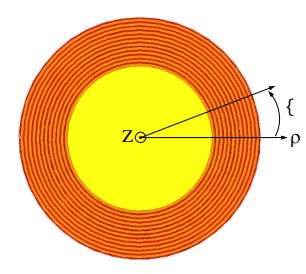

(b)
Figure 3: (a) The schematic of the single-turn air-lifted inductor using 1-port coplanar waveguide $(\mathrm{CPW})$ feeding, (b) the cross section of the wire including the $28 \mu \mathrm{m}$-diameter gold wire (yellow) as the core and 21 layers of $\mathrm{Cu}, \mathrm{NiFe}$ (orange, red) stack to realize the cylindrical radial superlattice (CRS) structure.

The CRS conductors have been analyzed by performing the full-wave simulations using the high frequency structure simulator (HFSS, ANSYS Inc.) that proves the effectiveness of the proposed method for conductor loss reduction. A $10 \mu \mathrm{m}$-length unit cell of the CRS conductor consists of a solid core conductor covered by laminated superlattice structures (Figure 1) where a ground plane is used underneath the structure, to allow propagation of transverse electromagnetic (TEM) waves. The lumped element equivalent circuit model, will be used to extract the conduction resistance of the conductor. Figure 4 shows the resistance of the conductor unit cell where $r=t_{\mathrm{Cu}} / \mathrm{t}_{\mathrm{NiFe}}=2.5$. The minimum resistance spectra occurs when $\mu_{\mathrm{eff}}=0$ which will be satisfied when $\mu_{\mathrm{NiFe}}=-2.5$ as expected in Figure 2. The simulation results reveal an ohmic loss reduction of about 3 times compared to the solid-core conductor at the target frequency of $15 \mathrm{GHz}$. For frequencies above $10 \mathrm{GHz}$, the resistivity of the superlattice structure falls below that of the solid-core conductor which leads to a relatively wide bandwidth where eddy current cancelling is effective.

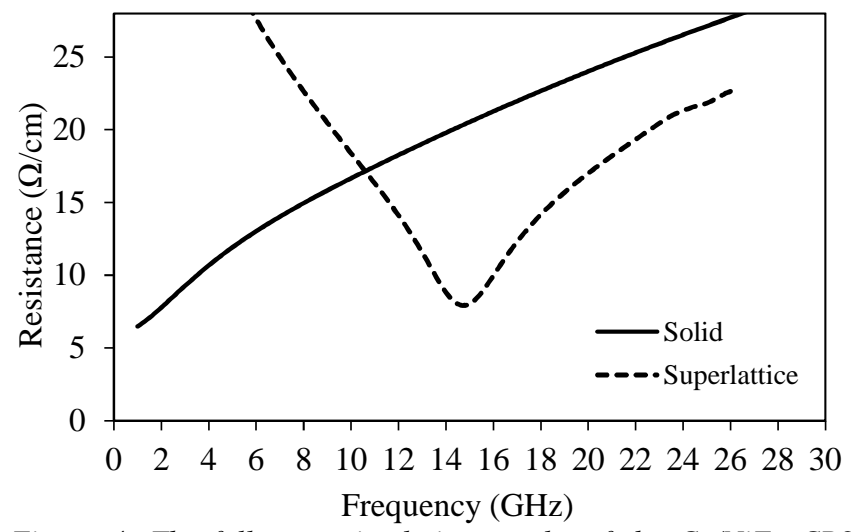

Figure 4: The full-wave simulation results of the $\mathrm{Cu} / \mathrm{NiFe} \mathrm{CRS}$ structures with $N=21$ and $t_{C u} / t_{N i F e}=400 \mathrm{~nm} / 160 \mathrm{~nm}$.

Figure 5 depicts the simulation results at $15 \mathrm{GHz}$ where the current distribution throughout the CRS conductor is showed (a) and compared with that of the regular conductor made of copper only (b) where a solid gold core with a diameter of $28 \mu \mathrm{m}$ has been used. Due to the more uniform current distribution at the operation frequency, the ohmic loss has been significantly reduced.

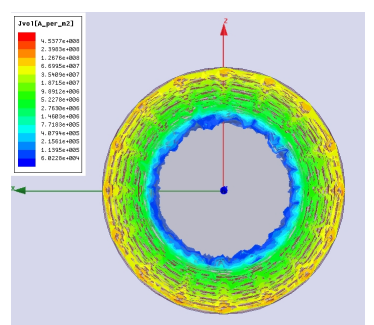

(a)

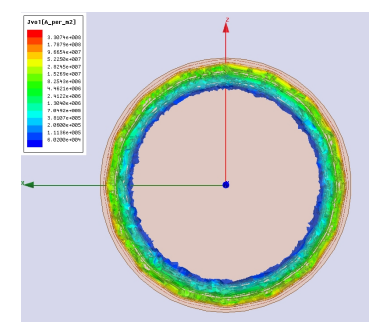

(b)
Figure 5: Current distribution of (a) the CRS conductor using a gold core of $28 \mu \mathrm{m}$ diameter, (b) the solid core conductor using single layer copper.

\section{FABRICATION AND EXPERIMENTAL RESULTS}

Thin film permalloy and copper are used as the magnetic and nonmagnetic metals, respectively, to create the CRS structure where both are electroplated using our in-house solution baths. The fabrication starts with a glass substrate; after the deposition of the seed layers $(\mathrm{Ti} / \mathrm{Cu} / \mathrm{Ti})$, the pads are patterned on the glass substrate followed by $10 \mu \mathrm{m}$ electroplating of $\mathrm{Cu}$. Then, the low-temperature gold wire bonding has been immediately performed in order to avoid oxidation of the copper and requirement of another metal layer on top of copper for the sake of wire bonding. Electroplating has been selected as the low cost, manufacturing method for the deposition of magnetic/nonmagnetic nanoscopic thin films on radial-shape gold core conductors to ensure conformal coating where most other processes including DC sputtering [7] would be more expensive and 
might not work best for the radial-shape devices. After performing the multiple-step electroplating of $\mathrm{Cu} / \mathrm{NiFe}$, the devices are released by etching the seed layers. Table 1 shows the utilized solution bath for the deposition of permalloy $\left(\mathrm{Ni}_{80} \mathrm{Fe}_{20}\right)$ thin films.

Table 1: The composition of permalloy $\left(\mathrm{Ni}_{80} \mathrm{Fe}_{20}\right)$ electrolyte

Compound (g/L)

\begin{tabular}{ll}
\hline \hline Nickel Sulfate & 200 \\
Iron Sulfate & 8 \\
Nickel Chloride & 5 \\
Boric Acid & 25 \\
Saccharin & 3 \\
$\mathrm{pH}$ & 4 \\
Temperature & $24^{\circ} \mathrm{C}$
\end{tabular}

Figure 6 shows the pictures of the fabricated single-turn airlifted CRS inductors. The inductors with the same pad size but different lengths are fabricated on the same substrate.
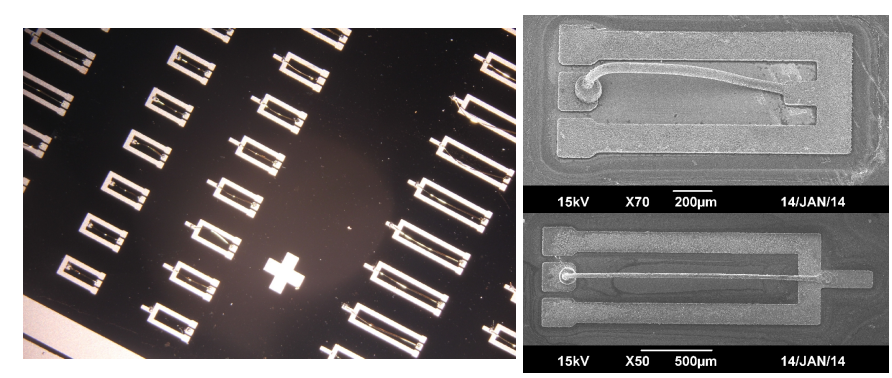

Figure 6: (a) the glass substrate holding the array of CRS inductors, and $(b)$ the CRS inductors of 1 and $2 \mathrm{~mm}$ length after electroplating. The backbone wire has been implemented using a gold wire bonder.

The solid core inductors are also fabricated using a single layer of copper with the same thickness as the superlattice multilayers. Figures 7 to 9 show the measurement results of the implemented CRS inductors where regular wire (solid core) inductors are also measured as control devices for comparison.

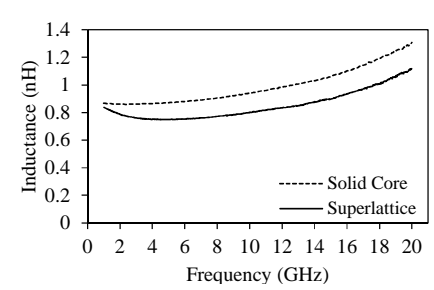

(a)

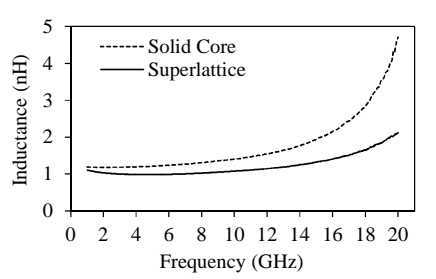

(b)
Figure 7: The measured inductance of superlattice and solid core inductors with the distance between the probe and the ground of (a) $1 \mathrm{~mm}$ and (b) $2 \mathrm{~mm}$.

The measurement results of two sets of inductors with different lengths but a conformal deposition of 21 layers of $\mathrm{NiFe} / \mathrm{Cu}(160$ $\mathrm{nm} / 400 \mathrm{~nm}$ ) layers are presented in this work. Multiple $\mathrm{Ni}$ and $\mathrm{Cu}$ targets facing the substrate inside the electroplating bath have been used for a uniform deposition of both $\mathrm{NiFe}$ and $\mathrm{Cu}$ around the radialshape inductors. The inductance and resistance of the devices are extracted after analyzing the one-port Y-parameters. The measured inductance values of the CRS and solid-core inductors are depicted in Figure 7. The inductance values of both of them are in the same range while the solid-core inductors show a slightly higher inductance values compared to CRS ones.
Figure 8 shows the measured quality factor (Q-factor) of the implemented inductors. As predicted in Figure 4, the ohmic resistance of the single layer copper constantly increases with the frequency due to the skin effect. The ohmic resistance of the CRS inductors is higher than that of the solid-wire inductors (Figure 4) in frequencies below $10 \mathrm{GHz}$; however, it starts to fall below the resistance of solid-wire inductors for frequencies above $10 \mathrm{GHz}$. Therefore, the Q-factor of the CRS inductors are higher compared to that of the solid-wire inductors in the operation frequency range of $10 \mathrm{GHz}<\mathrm{f}<20 \mathrm{GHz}$. Therefore, the proposed structure is a solution to the problem of high resistivity/low Q-factor of RF passive devices originating from the skin effect. By changing the $r$ $=\mathrm{t}_{\mathrm{Cu}} / \mathrm{t}_{\mathrm{NiFe}}$ ratio, the minimum resistance spectra point will be tuned, and that makes it possible to overpass the skin effect problem and design low loss passive devices operating in an intended operation frequency range.

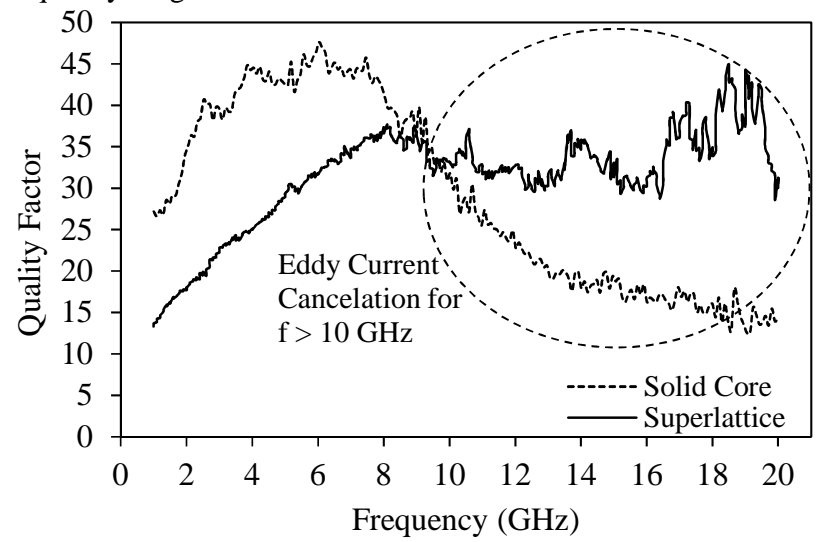

(a)

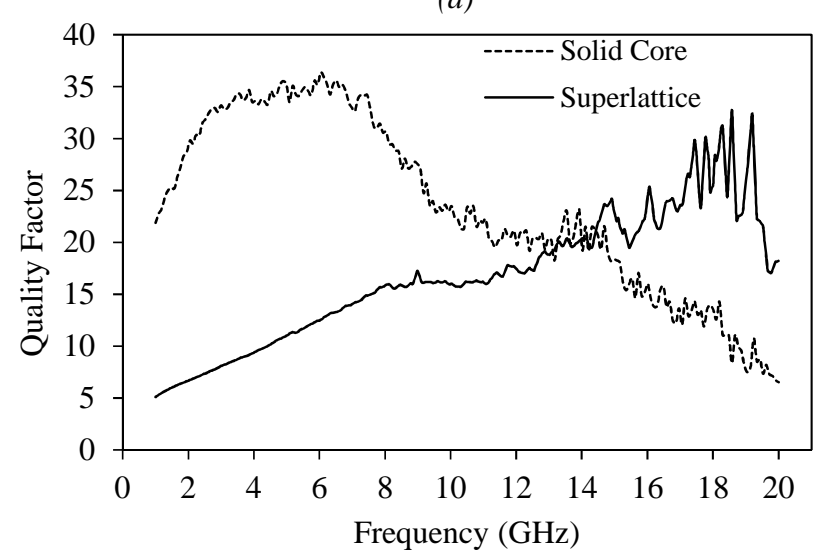

(b)

Figure 8: The measured $Q$-factors of the solid-wire and superlattice-wire inductors of (a) the 1-mm long inductor and (b) the 2-mm long inductor. As predicted in Figure 4, the resistance of the CRS device is lower than that of the solid-wire one in the range of 10-24 GHz. A higher Q-factor is achieved for $10-20 \mathrm{GHz}$ measurement for (a) and 14-20 GHzfor (b) proving the effectiveness of eddy current cancellation for the CRS devices. Using single-layer solid-wire devices, the $Q$-factor continuously decreases for the frequencies above $10 \mathrm{GHz}$.

Figure 9 shows a comparison of the measured resistance of the conductors of 1-mm and 2-mm length. The CRS conductors have higher resistance in low frequency range due to the high permeability of NiFe thin films in that frequency range which makes the skin depth smaller (equation (1)) and increases the ohmic loss. 
However, as the frequency increases, the total CRS resistance is kept below that of solid wire devices and a wide-band of operation is achieved.

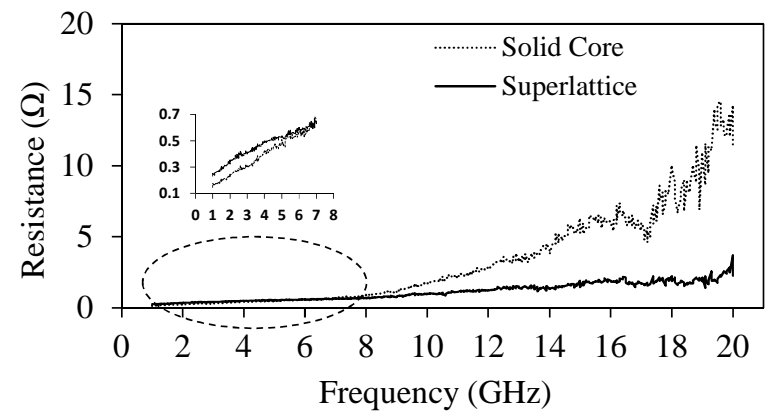

(a)

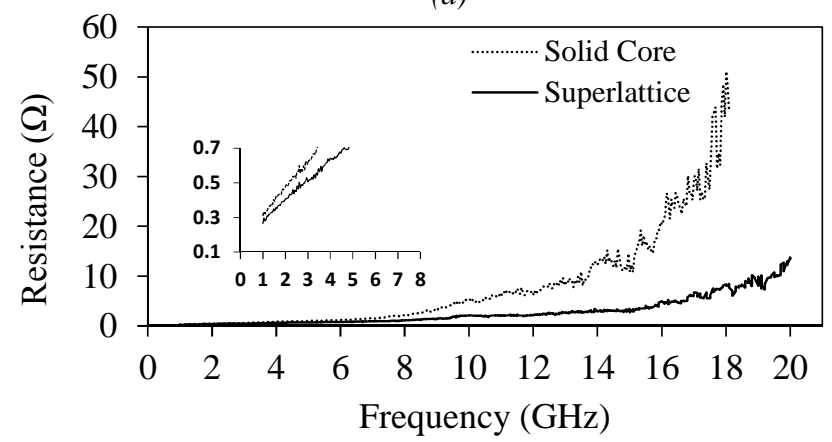

(b)

Figure 9: The measured resistance of the superlattice inductor and the solid wire inductor. The resistance is obtained based on the equivalent circuit model of the inductor.

\section{CONCLUSIONS}

In this paper, the experimental implementation of the CRS conductors and one of their potential applications as a high Q-factor inductor has been demonstrated. The proposed inductors benefit from the cylindrical radial shape of the conductors which inherently has a closed boundary condition in a radial direction with no fringing effects and therefore is considered more appropriate for eddy current suppression. The simulation and measurement results on the reduction of the conductor loss have been verified. The highest Qfactor $\mathrm{Ku}$ band inductor shows a Q-factor of 45 at $18 \mathrm{GHz}$, where the high Q-factor is attributed to the low dielectric loss from the airlifted architecture and the low conductor loss from the CRS conductor. Besides high Q-factor inductors shown in this work, the technology could be further extended to fabricate low loss passive devices including coaxial transmission lines and antennas. The fabrication method is fully compatible with the standard MEMS and CMOS processes and therefore can be integrated with the current manufacturing processes for reducing the RF loss. In this work, $\mathrm{NiFe}$ with $\mathrm{f}_{\mathrm{AR}}=28 \mathrm{GHz}$ has been used which limits the maximum frequency of operation to be smaller than $f_{A R}$; however, other ferromagnetic materials with higher $f_{A R}$ could be used to design low loss passive devices in higher frequency ranges [8-9].

\section{ACKNOWLEDGMENTS}

This work is supported in part by NSF ECCS (1132413). Microfabrication and magnetic characterization have been performed in Nanoscale Research Facilities (NRF) cleanroom and Interdisciplinary Microsystems Group (IMG), at the University of Florida.

\section{REFERENCES}

[1] B. Pan, Y. Li, M. M. Tentzeris, and J. Papapolymerou, "A novel low-loss integrated $60 \mathrm{GHz}$ cavity filter with source-load coupling using surface micromachining technology," Microwave Symposium Digest, IEEE MTT-S International, pp.639-642, Jun. 2008.

[2] Y.K. Yoon, J.W. Park, and M. G. Allen, "Polymer-core conductor approaches for RF MEMS," IEEE Journal of Microelectromechanical Systems, vol. 14, no. 5, pp. 886-894, Oct. 2005.

[3] B. Rejaei and M. Vroubel, "Suppression of skin effect in metal/ferromagnet superlattice conductors," Journal of Applied physics, vol. 96, no.11, pp. 6863-6868, 2004.

[4] J. Wu and Y.K. Yoon, "A Low Ohmic Loss Radial Superlattice Conductor at $15 \mathrm{GHz}$ using Eddy Current Canceling Effect," IEEE Antenna and Propagation Society International Symp. (APS/URSI), pp. 1552 - 1553, Jul. 2013.

[5] J. B. Youssef, N. Vukadinovic, D. Billet, and M. Labrune "Thickness-dependent magnetic excitations in permalloy films with nonuniform magnetization," Physical Review B 69.17, 2004.

[6] Y. Liu, L. Chen, C. Y. Tan, H. J. Liu, and C. K. Ong, "Broadband complex permeability characterization of magnetic thin films using shorted microstrip transmission-line perturbation," Review of Scientific Instruments, 76.6, 063911, 2005.

[7] I. Iramnaaz, H. Schellevis , B. Rejaei , R. Fitch , and Y. Zhuang, "Self-Biased Low Loss Conductor Featured With Skin Effect Suppression for High Quality RF Passives," IEEE Transactions on Magnetics, vol. 48, no.11, pp. 4139-4142, Nov. 2012.

[8] Y. Liu, C. Y. Tan, Z. W. Liu, and C. K. Ong "FeCoSiN film with ordered FeCo nanoparticles embedded in a Si-rich matrix," Applied physics letters, 90, 112506, 2007.

[9] A. Rahimi and Y.K. Yoon, "Low Loss Conductors for CMOS and Through Glass/Silicon Via (TGV/TSV) Structures Using Eddy Current Cancelling Superlattice Structure," proceedings of IEEE Electronic Components and Technology Conference (ECTC), Jun. 2014.

\section{CONTACT}

*A. Rahimi: arahimi@ufl.edu

YK Yoon: ykyoon@ece.ufl.edu 